\title{
Remarks and questions on the article "Prospective randomized double-blind trial of racecadotril compared with loperamide in elderly people with gastroenteritis living in nursing homes"
}

\author{
Robert Hofmann
}

Received: 16 April 2010 /Accepted: 12 May 2010 /Published online: 5 June 2010

(C) Springer-Verlag 2010

The article "Prospective randomized double-blind trial of racecadotril compared with loperamide in elderly people with gastroenteritis living in nursing homes" by Gallelli et al. [1] raises a lot of concerns i.a.:

1. Despite an intensive search the study could not be located in any registry, which means the protocol was not accessible. The author did not provide any information.

2. The study is said to be "double-blind"; however, the posology of racecadotril and loperamide are completely different with regard to their starting dose and intervals. No double dummy technique is reported. At best it is single-blind; however, people of that age usually already have pre-experience with loperamide so even this is not certain.

3. No hypothesis is reported; therefore, no power calculation of cases needed was performed (and reported) and no statistical plan reported.

4. The pricing is calculated on five tablets, although the study limits racecadotril to three and loperamide to four.

5. As a primary efficacy criterion "duration of diarrhea in days" is named. There is no information on how diarrhea was defined, how the end of diarrhea was defined (Time to last unformed stool? Time to first formed stool?).

R. Hofmann $(\bowtie)$

Johnson\&Johnson, Medical Affairs,

Neuss, Germany

e-mail: rhofmann@its.jnj.com

URL: www.jnj.com
6. "In about $50 \%$ of patients (age $80 \pm 0.2$ years; range $73-$ 93 years), in the loperamide group during the study period, 4 days of treatment were ineffective"; about $50 \%$ comprises 16 or 17 patients. Even if all but 2 were exactly 80 and the 2 were 73 and 93 years of age, the standard deviation would not be 0.2 but 3.7 .

7. It is completely implausible how in $50 \%$ of loperamide patients the treatment was ineffective for 4 days, but at the same time $60 \%$ had constipation as a side effect. If you are constipated you don't have diarrhea!

8. How was the pharmacoeconomic evaluation performed? The direct drug cost was $1.65 €$ per day for loperamide and $1.725 €$ for racecadotril. The study was limited to 4 days. How were the numbers of $107.5 €$ and $44.85 €$ calculated? In the discussion, again, other higher numbers are introduced.

I wonder how the authors could draw their conclusions from a non-double-blind study that is likely to be underpowered, with elusive, partly erroneous reporting. Writing them into the headline and abstract leads to wide uncritical reference.

\section{Reference}

1. Gallelli L, Colosimo M, Tolotta GA, Falcone D, Luberto L, Curto LS, Rende P, Mazzei F, Marigliano NM, De Sarro G, Cucchiara S (2010) Prospective randomized double-blind trial of racecadotril compared with loperamidein elderly people with gastroenteritis living in nursing homes. Eur J Clin Pharmacol 66(2):137-144 